AIAA 2001-4273

\title{
INTEGRATED ORBIT, ATTITUDE, AND STRUCTURAL CONTROL SYSTEM DESIGN FOR SPACE SOLAR POWER SATELLITES
}

\author{
Bong Wie* \\ Arizona State University \\ Tempe, Arizona 85287-6106
}

\begin{abstract}
The major objective of this study is to develop an integrated orbit, attitude, and structural control system architecture for very large Space Solar Power Satellites (SSPS) in geosynchronous orbit. This study focuses on the 1.2-GW "Abacus" SSPS concept characterized by a $3.2 \times 3.2 \mathrm{~km}$ solar-array platform, a 500-m diameter microwave beam transmitting antenna, and a $500 \times 700 \mathrm{~m}$ earth-tracking reflector. For this baseline Abacus SSPS configuration, we derive and analyze a complete set of mathematical models, including external disturbances such as solar radiation pressure, microwave radiation, gravity-gradient torque, and other orbit perturbation effects. The proposed control system architecture utilizes a minimum of $5001-\mathrm{N}$ electric thrusters to counter, simultaneously, the cyclic pitch gravity-gradient torque, the secular roll torque caused by an offset of the centerof-mass and center-of-pressure, the cyclic roll/yaw microwave radiation torque, and the solar radiation pressure force whose average value is about $60 \mathrm{~N}$.
\end{abstract}

\section{Introduction}

A renewed interest in space solar power is spurring a reexamination of the prospects for generating large amounts of electricity from large-scale, space-based solar power systems. Peter Glaser (Refs. 1-2) first proposed the Satellite Solar Power Station (SSPS) concept in 1968 and received a U.S. patent on a conceptual design for such a satellite in 1973. As a result of a series of technical and economic feasibility studies by NASA and Department of Energy in the 1970s, an SSPS reference system was developed in the late 1970s. The 1979 SSPS reference system, as it is called, featured a very large solar array platform $(5.3 \times 10.7 \mathrm{~km})$ and a double-gimballed microwave beam transmitting antenna

*Professor, Dept. of Mechanical \& Aerospace Engineering, (480) 965-8674, bong.wie@asu.edu, Associate Fellow AIAA.

$\dagger$ Aerospace Engineer, c.m.roithmayr@larc.nasa.gov, (757) 8646778, Senior Member AIAA. Copyright (C)2001 by the American Institute of Aeronautics and Astronautics, Inc. All rights reserved.

\author{
Carlos Roithmayr ${ }^{\dagger}$ \\ NASA Langley Research Center \\ Hampton, Virginia 23681-2199
}

( $1 \mathrm{~km}$ diameter). The total mass was estimated to be $50 \times 10^{6} \mathrm{~kg}$. A ground or ocean-based rectenna (rectifying antenna) measuring $10 \times 13 \mathrm{~km}$ would receive the microwave beam on the earth and deliver up to $5 \mathrm{GW}$ of electricity.

In 1995, NASA revisited the Space Solar Power (SSP) concept to assess whether SSP-related technologies had advanced enough to alter significantly the outlook on the economic and technical feasibility of space solar power. The "Fresh Look" study (Ref. 3), conducted by NASA during 1995-1997, found that in fact a great deal had changed and that multi-megawatt SSP satellites appear viable, with strong space applications. The study also found that ambitious research, technology development and validation over a period of perhaps 15-20 years are required to enable SSP concepts to be considered "ready" for commercial development.

Recent studies by NASA as part of the SSP Exploratory Research and Technology (SERT) program have produced a variety of new configurations of Space Solar Power Satellites (SSPS), including the "Abacus" configuration, as described in Refs. 4-6. Some of these configurations, such as the "Sun Tower" configuration, are based on the passive gravity-gradient stabilization concept. However, most other configurations require three-axis attitude control to maintain continuous sun tracking of the solar arrays in the presence of external disturbances including the gravity-gradient torque. A cylindrical configuration, which is not affected by the troublesome pitch gravity-gradient torque, has also been considered by NASA (Ref. 6).

This study focuses on the 1.2-GW "Abacus" satellite configuration shown in Figure 1. This Abacus satellite is characterized by its simple configuration consisting of an inertially oriented, $3.2 \times 3.2 \mathrm{~km}$ solar-array platform, a 500-m diameter microwave beam transmitting antenna fixed to the platform, and a $500 \times 700 \mathrm{~m}$ rotating reflector that tracks the earth. Some unique features of the Abacus satellite relative to the 1979 SSPS reference system are:

- The transmitting antenna is not gimballed; instead, 


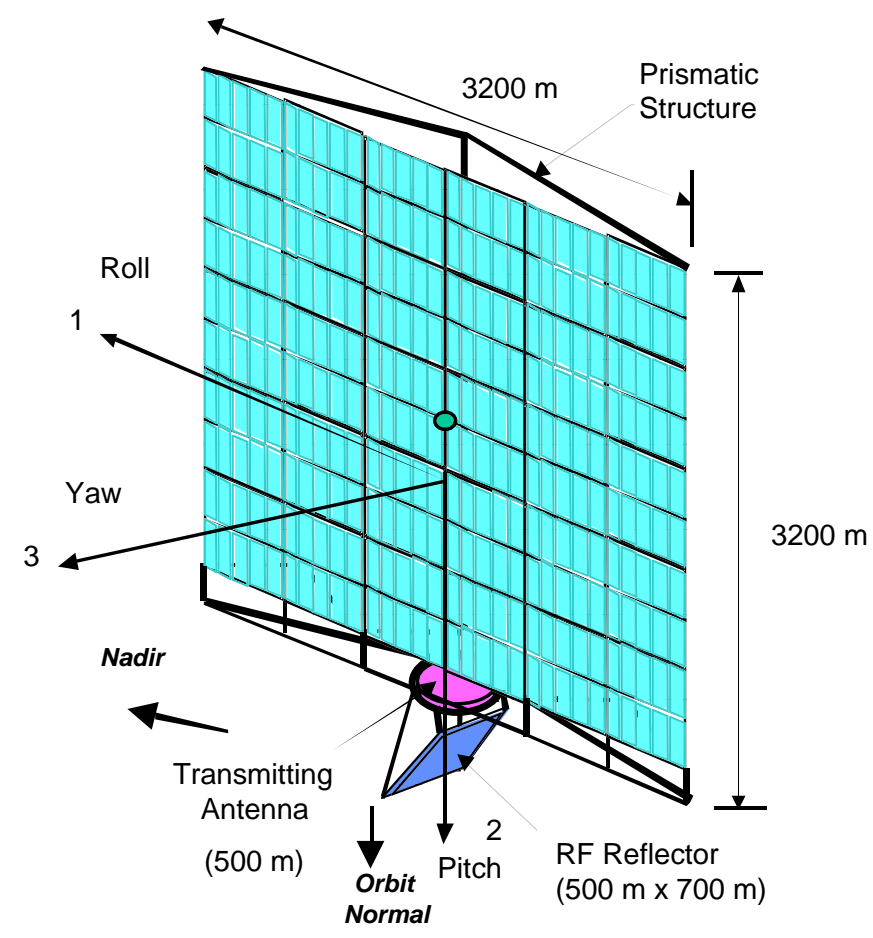

Figure 1: Baseline 1.2-GW "Abacus" satellite configuration (Refs. 4-6).

an azimuth roll-ring mounted, rotating reflector provides earth pointing of the microwave beam.

- The rotating reflector design thus eliminates massive rotary joint and slip rings of the 1979 SSPS reference system.

- Links activated by ball-screw mechanisms tilt the reflector to point to ground stations at various latitudes

The objectives of this study, in support of the SERT program of NASA, are: (i) to develop preliminary concepts for orbit, attitude, and structural control of very large SSPS using a variety of actuators such as control moment gyros, momentum wheels, and electric propulsion thrusters; (ii) to develop mathematical models, define a top-level control system architecture, and perform control system design and analysis for a baseline Abacus satellite configuration in geosynchronous orbit; and (iii) to determine the required number, size, placement, mass, and power for the actuators to control the orbit, attitude, and structural motions of the baseline Abacus satellite.

\section{Mass Properties, Disturbances, and Control Requirements}

\subsection{Geometric Properties}

The three major parts of the Abacus satellite and their dimensions are shown in Figure 1; the mass of each part
Table 1: Geometric and mass properties of the 1.2-GW Abacus satellite

\begin{tabular}{ll}
\hline Solar array mass & $21 \times 10^{6} \mathrm{~kg}$ \\
Transmitting antenna mass & $3 \times 10^{6} \mathrm{~kg}$ \\
Reflector mass & $0.8 \times 10^{6} \mathrm{~kg}$ \\
Total mass & $\mathrm{m}=25 \times 10^{6} \mathrm{~kg}$ \\
Platform area & $\mathrm{A}=3200 \mathrm{~m} \times 3200 \mathrm{~m}$ \\
Area-to-mass ratio & $\mathrm{A} / \mathrm{m}=0.4 \mathrm{~m}^{2} / \mathrm{kg}$ \\
Roll inertia & $J_{1}=2.8 \times 10^{13} \mathrm{~kg}-\mathrm{m}^{2}$ \\
Pitch inertia & $J_{2}=1.8 \times 10^{13} \mathrm{~kg}-\mathrm{m}^{2}$ \\
Yaw inertia & $J_{3}=4.6 \times 10^{13} \mathrm{~kg}-\mathrm{m}^{2}$ \\
cm-cp offset & $200 \mathrm{~m}$ (along pitch axis) \\
cm-cp offset (uncertainty) & $\pm 20 \mathrm{~m}$ (along roll axis) \\
\hline
\end{tabular}

Table 2: Solar pressure and microwave radiation disturbances

\begin{tabular}{ll}
\hline Solar pressure force & $(4.5 \mathrm{E}-6)(1.3)(\mathrm{A})=60 \mathrm{~N}$ \\
Solar pressure torque (roll) & $60 \mathrm{~N} \times 200 \mathrm{~m}$ \\
Solar pressure torque (pitch) & $60 \mathrm{~N} \times 20 \mathrm{~m}$ \\
Reflector radiation force & $7 \mathrm{~N} \mathrm{(rotating} \mathrm{force)}$ \\
Reflector radiation torque & $7 \mathrm{~N} \times 1700 \mathrm{~m}$ \\
\hline
\end{tabular}

is given in Table 1, together with the total mass and area of the spacecraft. The mass of the reflector is approximately $3 \%$ of the total mass; therefore, the reflector's mass can be neglected in the analysis of attitude motion, simplifying the task in two important respects. First, the Abacus satellite can be treated as a single body rather than a multibody spacecraft. When the Abacus satellite is regarded as rigid, the spacecraft's moments and products of inertia for a set of axes fixed in the solar array do not vary with time. Second, when the unsymmetrical mass distribution of the reflector is left out of account, the principal axes of inertia of the spacecraft with respect to the spacecraft's mass center are parallel to the roll, pitch, and yaw axes illustrated in Figure 1. The moments of inertia for these axes, henceforth considered to be principal moments of inertia, are given in Table 1. The center of pressure is located $100 \mathrm{~m}$ below the geometric center of the square platform, the center of mass is located $300 \mathrm{~m}$ below the geometric center along the pitch axis, and $\pm 20 \%$ overall uncertainty in the mass properties should be considered in control design.

\subsection{External Disturbances}

External disturbances acting on the Abacus satellite include: solar radiation pressure force, microwave radiation force, gravity-gradient torque, and other orbit perturbation forces. Some of these disturbances with $\pm 20 \%$ overall uncertainties are summarized in Table 2. Disturbance torques in units of $\mathrm{N}-\mathrm{m}$, due to solar pressure, 
Table 3: Orbit parameters and control requirements

\begin{tabular}{ll}
\hline Earth's gravitational parameter & $\mu=398,601 \mathrm{~km}^{3} / \mathrm{s}^{2}$ \\
Geosynchronous orbit $(e, i \approx 0)$ & $a=42,164 \mathrm{~km}$ \\
Orbit period & $23 \mathrm{hr} 56 \mathrm{~min} 4 \mathrm{sec}$ \\
Orbit rate & $n=7.292 \times 10^{-5} \mathrm{rad} / \mathrm{sec}$ \\
Stationkeeping accuracy & $\pm 0.1 \mathrm{deg}$ \\
Solar array pointing accuracy & $\pm 0.5 \mathrm{deg}$ for roll $/ \mathrm{pitch}$ \\
Microwave beam pointing accuracy & $\pm 5 \mathrm{arcmin}$ \\
\hline
\end{tabular}

Table 4: A large single-gimbal CMG

\begin{tabular}{ll}
\hline Cost & $\$ 1 \mathrm{M}$ \\
Momentum & $7,000 \mathrm{~N}-\mathrm{m}-\mathrm{s}$ \\
Max torque & $4,000 \mathrm{~N}-\mathrm{m}$ \\
Peak power & $500 \mathrm{~W}$ \\
Mass & $250 \mathrm{~kg}$ \\
Momentum/mass & $28 \mathrm{~N}-\mathrm{m}-\mathrm{s} / \mathrm{kg}$ \\
\hline
\end{tabular}

microwave radiation, cm-cp offset, and cm-cp offset uncertainty, can be expressed along the platform-fixed control axes as:

$$
\begin{aligned}
\text { Roll: } & d_{1} \approx 12,000-11,900 \cos n t \\
\text { Pitch: } & d_{2} \approx 1200 \\
\text { Yaw: } & d_{3} \approx-11,900 \sin n t
\end{aligned}
$$

where $n$ is the orbital rate of the Abacus satellite and $t$ is time. The constant pitch disturbance torque of 1200 $\mathrm{N}-\mathrm{m}$ is due to the assumed cm-cp offset of $20 \mathrm{~m}$ along the roll axis, and $\pm 20 \%$ uncertainty in this disturbance model should also be considered in control design. In addition to these disturbances, gravity-gradient disturbance torques are also acting on the Abacus satellite. It is assumed that the electric currents circulate in the solar array structure in such a way that magnetic fields cancel out and the Abacus satellite is not affected by the magnetic field of the earth.

\subsection{Orbit Parameters and Control Re- quirements}

Basic orbital characteristics and control requirements for the Abacus satellite in geosynchronous orbit are summarized in Table 3.

\section{Technical Issues}

\subsection{Momentum Storage Requirement}

Assuming that the gravity gradient torque is the only external disturbance torque acting along the pitch axis, we consider the pitch equation of motion of a rigid spacecraft in geosynchronous orbit given by

$$
J_{2} \ddot{\theta}_{2}=\frac{3 n^{2}}{2}\left(J_{3}-J_{1}\right) \sin 2 \theta_{2}+u_{2}
$$

where $J_{1}, J_{2}$, and $J_{3}$ are, respectively, the roll, pitch, and yaw principal moments of inertia; $\theta_{2}$ is the pitch angle measured from the LVLH (local vertical and local horizontal) reference frame; $n$ is the orbit rate; and $u_{2}$ is the pitch control torque.

For continuous sun pointing of the Abacus platform with $\theta_{2}=n t$, the pitch control torque required to counter the cyclic gravity-gradient torque simply becomes

$$
u_{2}=-\frac{3 n^{2}}{2}\left(J_{3}-J_{1}\right) \sin 2 n t
$$

with peak values of $\pm 143,000 \mathrm{~N}-\mathrm{m}$. If angular momentum exchange devices, such as momentum wheels (MWs) or control moment gyros (CMGs), are to be employed for pitch control, the peak angular momentum to be stored can then be estimated as

$$
H_{\max }=\frac{3 n}{2}\left(J_{3}-J_{1}\right)=2 \times 10^{9} \mathrm{~N}-\mathrm{m}-\mathrm{s}
$$

This is is about 100,000 times the angular momentum storage requirement of the International Space Station (ISS). The ISS is controlled by four double-gimballed CMGs with a total momentum storage capability of about 20,000 N-m-s. The double-gimballed CMGs employed by the ISS have a momentum density of $17.5 \mathrm{~N}$ $\mathrm{m}-\mathrm{s} / \mathrm{kg}$, and future advanced flywheels may have a larger momentum density of $150 \mathrm{~N}-\mathrm{m}-\mathrm{s} / \mathrm{kg}$. Basic characteristics of a large single-gimbal CMG are also summarized in Table 4.

Based on the preceding discussion, it can be concluded that a traditional momentum management approach using conventional CMGs (or even employing future advanced flywheels) is not a viable option for controlling very large Space Solar Power Satellites.

To meet the momentum storage requirement of very large SSPS, a concept of constructing large-diameter momentum wheels in space has been studied in the late 1970s (Ref. 7). In an attempt to resolve the angular momentum storage problem of large sun-pointing spacecraft, a quasi-inertial sun-pointing, pitch control concept was also developed by Elrod in 1972 (Ref. 8), and further investigated by Juang and Wang in 1982 (Ref. 9). However, such a "free-drift" concept is not a viable option for the Abacus satellite because of the large pitch attitude peak error of $18.8 \mathrm{deg}$ and its inherent sensitivity with respect to initial phasing and other orbital perturbations.

Because the pitch gravity-gradient torque becomes naturally zero for cylindrical, spherical or beam-like satellites with $J_{1}=J_{3}$, a cylindrical SSPS configuration was also studied by NASA to simply avoid such a troublesome pitch gravity-gradient torque problem. 


\section{Solar pressure constant $\mathrm{P}$}

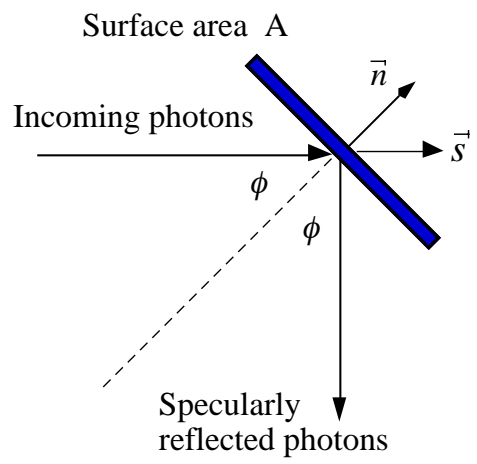

Figure 2: Solar radiation pressure force acting on an ideal flat surface.

\subsection{Solar Radiation Pressure and Large Area-to-Mass Ratio}

Despite the importance of the cyclic pitch gravitygradient torque, this study shows that the solar radiation pressure force is considerably more detrimental to control of the Abacus satellite (and also other large SSPS) because of an area-to-mass ratio that is very large compared to contemporary, higher-density spacecraft.

The significant orbit perturbation effect of the solar radiation pressure on large spacecraft with large area-tomass ratios has been investigated by many researchers in the past. A detailed physical description of the solar radiation pressure can be found in a recent book on solar sailing by McInnes (Ref. 10).

The solar radiation pressure forces are due to photons impinging on a surface in space, as illustrated in Figure 2. Assuming that a fraction, $\rho_{s}$, of the impinging photons is specularly reflected, a fraction, $\rho_{d}$, is diffusely reflected, and a fraction, $\rho_{a}$, is absorbed by the surface, we have

$$
\rho_{s}+\rho_{d}+\rho_{a}=1
$$

The solar radiation pressure (SRP) force acting on an ideal flat surface is then expressed as

$$
\vec{F}=P A(\vec{n} \cdot \vec{s})\left\{\left(\rho_{a}+\rho_{d}\right) \vec{s}+\left(2 \rho_{s}(\vec{n} \cdot \vec{s})+\frac{2}{3} \rho_{d}\right) \vec{n}\right\}
$$

where $P=4.5 \times 10^{-6} \mathrm{~N} / \mathrm{m}^{2}$ is the nominal solar radiation pressure constant, $A$ is the surface area, $\vec{n}$ is a unit vector normal to the surface, and $\vec{s}$ is a unit vector pointing from the sun to satellite.

For an ideal case of a perfect mirror with $\rho_{d}=\rho_{a}=0$ and $\rho_{s}=1$, we have

$$
\vec{F}=2 P A \cos ^{2} \phi \vec{n}
$$

where $A \cos \phi$ is called the projected area of the surface under consideration. Also for an ideal case of a black body with $\rho_{s}=\rho_{d}=0$ and $\rho_{a}=1$, we have

$$
\vec{F}=P A \cos \phi \vec{s}
$$

For most practical cases of satellites with small angles of $\phi$, the SRP perturbation force per unit mass is simply modeled as

$$
\vec{f}=P(1+\rho)(A / m) \vec{s}
$$

where $\rho$ is the overall surface reflectance ( 0 for a black body and 1 for a mirror) and $A / m$ is the area-to-mass ratio.

Let the SRP perturbation acceleration be expressed as

$$
\vec{f}=f_{r} \vec{e}_{r}+f_{\theta} \vec{e}_{\theta}+f_{z} \vec{e}_{z}
$$

where $\left\{\vec{e}_{r}, \vec{e}_{\theta}, \vec{e}_{z}\right\}$ is a set of unit vectors of the so-called perifocal reference frame. Ignoring the effects of seasonal variations of the sun vector, we simply obtain $f_{r} \approx f \sin \theta$ and $f_{\theta} \approx f \cos \theta$ where $f=P(1+\rho)(A / m)$ and $\theta$ is the true anomaly.

From the orbit perturbation analysis (Refs. 11-12), we have

$$
\begin{aligned}
\frac{d a}{d t} & =\frac{2}{n \sqrt{1-e^{2}}}\left\{f_{r} e \sin \theta+f_{\theta}(1+e \cos \theta)\right\} \\
\frac{d e}{d t} & =\frac{\sqrt{1-e^{2}}}{n a}\left\{f_{r} \sin \theta+f_{\theta}(\cos \theta+\cos E)\right\}
\end{aligned}
$$

where $\theta$ and $E$ are the true and eccentric anomalies, respectively. For geosynchronous satellites with $e \approx 0$, we obtain

$$
\begin{aligned}
& \frac{d a}{d t}=\frac{2}{n} f_{\theta}=\frac{2 f}{n} \sin \theta \\
& \Rightarrow \Delta a=0 \text { per day }
\end{aligned}
$$

and

$$
\begin{aligned}
\frac{d e}{d t} & =\frac{1}{n a}\left(f_{r} \sin \theta+2 f_{\theta} \cos \theta\right) \\
& =\frac{1}{n a}\left(f \sin ^{2} \theta+2 f \cos ^{2} \theta\right) \\
& =\frac{f}{n a}\left(\frac{3}{2}+\frac{1}{2} \cos 2 \theta\right) \\
\Rightarrow & \Delta e \approx \frac{3 \pi f}{n^{2} a} \text { per day }
\end{aligned}
$$

The solar radiation pressure effect on the longitude change can also be found as

$$
\begin{aligned}
\ddot{\lambda} & =\frac{d n}{d t}=-\frac{3 n}{2 a} \frac{d a}{d t}=-\frac{3 n}{2 a} \frac{2}{n} f_{\theta}=-\frac{3}{a} f_{\theta} \\
& =-\frac{3 f}{a} \cos \theta
\end{aligned}
$$

For the Abacus satellite, we have

Area-to-mass ratio $A / m \approx 0.4 \mathrm{~m}^{2} / \mathrm{kg}$

$\mathrm{SRP}$ force $\approx 60 \mathrm{~N}$

$\mathrm{SRP}$ perturbation acceleration $\approx 2.4 \times 10^{-6} \mathrm{~m} / \mathrm{s}^{2}$

$\Delta e=\frac{3 \pi\left(4.5 \times 10^{-6}\right)(1.3) A / m}{n^{2} a} \approx 1 \times 10^{-4}$ per day

$\Rightarrow$ Longitude drift $\Delta \lambda=2 \Delta e \approx 0.0115 \mathrm{deg} /$ day

$\Rightarrow$ maximum $\Delta e \approx 0.018$ at the mid year

$\Rightarrow \operatorname{maximum} \Delta \lambda=2 \Delta e \approx 2 \mathrm{deg} ; \operatorname{maximum} \Delta a \approx 1.8 \mathrm{~km}$ 

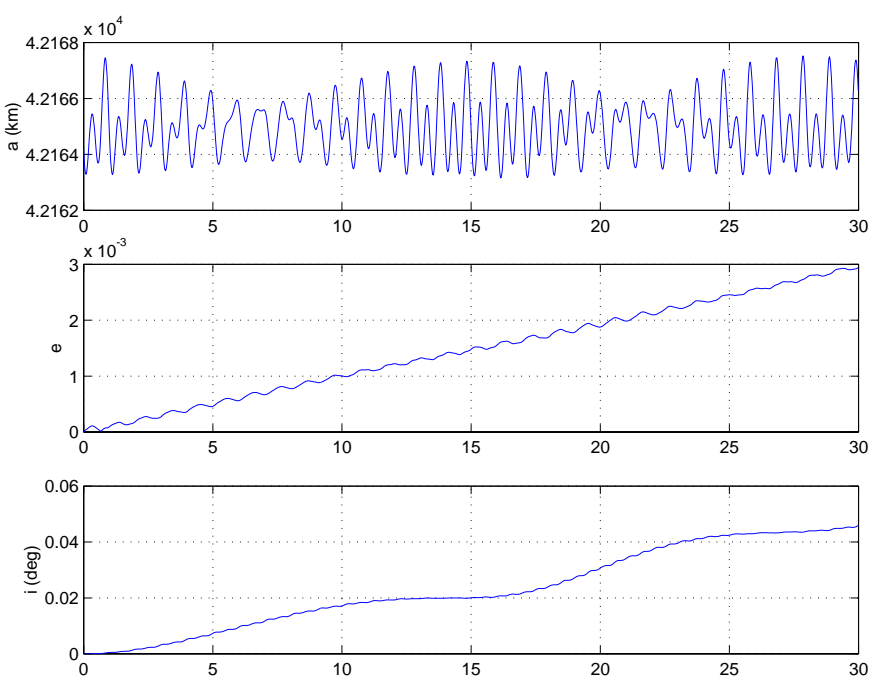

Figure 3: Orbit simulation results of the Abacus satellite with the effects of the earth's oblateness and triaxiality, luni-solar perturbations, and 60-N solar radiation pressure force (time in units of orbits).

Consequently, orbit eccentricity control using high- $I_{s p}$ ion engines becomes necessary. The yearly propellant requirement to counter the approximately $60-\mathrm{N}$ solar pressure force can be estimated as

$$
\Delta m=\frac{(60)(24 \times 3600 \times 365)}{5000 \times 9.8} \approx 40,000 \mathrm{~kg} / \text { year }
$$

where an ion engine with $I_{s p}$ of $5000 \mathrm{sec}$ is assumed. Detailed discussions of an electric propulsion system proposed for the Abacus satellite will be presented later in this paper.

Typical north-south and east-west stationkeeping maneuvers for the Abacus satellite will also require

$$
\Delta m=m\left(1-\exp \left(-\frac{\Delta V}{g I_{s p}}\right)\right) \approx 30,000 \mathrm{~kg} / \text { year }
$$

where $m=25 \times 10^{6} \mathrm{~kg}, \Delta V=50 \mathrm{~m} / \mathrm{s}$ per year, $g=9.8$ $\mathrm{m} / \mathrm{s}^{2}$, and $I_{s p}=5000 \mathrm{sec}$.

The results of 30-day simulations of orbital motion of the Abacus satellite, with the effects of the earth's oblateness and triaxiality, luni-solar perturbations, and 60-N solar pressure force included, are shown in Figure 3. It is worth noting the extent to which eccentricity and inclination are perturbed.

The initial values used in the simulations correspond to a circular, equatorial orbit of radius 42164.169 $\mathrm{km}$; therefore, the initial orbital elements are: $a=$ $42164.169 \mathrm{~km}$ and $e=i=\Omega=\omega=0$. The epoch used to calculate the solar and lunar positions, as well as the earth's orientation in inertial space, is March 21, 2000. In order to place the spacecraft at an initial terrestrial longitude of $75.07 \mathrm{deg}$ (one of the stable longitudes), a true anomaly $\theta$ of $253.89 \mathrm{deg}$ is used. These elements

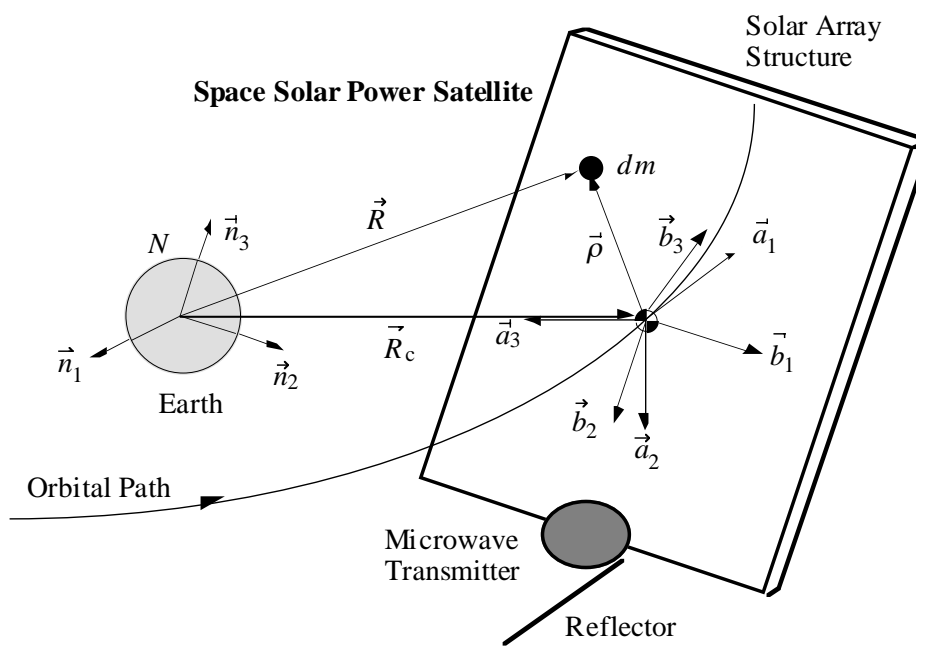

Figure 4: A large Space Solar Power Satellite (SSPS) in geosynchronous orbit.

correspond to an initial position and velocity of

$\vec{r}=X \vec{I}+Y \vec{J}+Z \vec{K}=-11698.237 \vec{I}-40508.869 \vec{J}(\mathrm{~km})$

$\vec{v}=2.954 \vec{I}-0.853 \vec{J}(\mathrm{~km} / \mathrm{s})$

where $\{\vec{I}, \vec{J}, \vec{K}\}$ is a set of unit vectors of the EarthCentered-Inertial (ECI) reference frame.

In the next section, we develop an attitude dynamics model of sun-pointing spacecraft in geosynchronous orbit for attitude control system architecture design.

\section{Attitude Equations of Motion of Sun-Pointing Spacecraft}

Consider a spacecraft in circular orbit, as illustrated in Figure 4. A local vertical and local horizontal (LVLH) reference frame $A$ with its origin at the center of mass of an orbiting spacecraft has a set of unit vectors $\left\{\vec{a}_{1}, \vec{a}_{2}, \vec{a}_{3}\right\}$ with $\vec{a}_{1}$ along the orbit direction, $\vec{a}_{2}$ perpendicular to the orbit plane, and $\vec{a}_{3}$ toward the earth, as illustrated in Figure 4. The angular velocity of $A$ with respect to an inertial or Newtonian reference frame $N$ is

$$
\vec{\omega}^{A / N}=-n \vec{a}_{2}
$$

where $n$ is the constant orbital rate. The angular velocity of the body-fixed reference frame $B$ with basis vectors $\left\{\vec{b}_{1}, \vec{b}_{2}, \vec{b}_{3}\right\}$ is then given by

$$
\vec{\omega}^{B / N}=\vec{\omega}^{B / A}+\vec{\omega}^{A / N}=\vec{\omega}^{B / A}-n \vec{a}_{2}
$$

where $\vec{\omega}^{B / A}$ is the angular velocity of $B$ relative to $A$.

To describe the orientation of the body-fixed reference frame $B$ with respect to the LVLH reference frame $A$ in terms of three Euler angles $\theta_{i}(i=1,2,3)$, consider the sequence of $\mathbf{C}_{1}\left(\theta_{1}\right) \leftarrow \mathbf{C}_{3}\left(\theta_{3}\right) \leftarrow \mathbf{C}_{2}\left(\theta_{2}\right)$ from the LVLH reference frame $A$ to a body-fixed reference frame $B$. 
For this rotational sequence, we have

$$
\left[\begin{array}{l}
\vec{b}_{1} \\
\vec{b}_{2} \\
\vec{b}_{3}
\end{array}\right]=\left[\begin{array}{lll}
C_{11} & C_{12} & C_{13} \\
C_{21} & C_{22} & C_{23} \\
C_{31} & C_{32} & C_{33}
\end{array}\right]\left[\begin{array}{l}
\vec{a}_{1} \\
\vec{a}_{2} \\
\vec{a}_{3}
\end{array}\right]
$$

where $C_{11}=\cos \theta_{2} \cos \theta_{3}, C_{12}=\sin \theta_{3}, C_{13}=$ $-\sin \theta_{2} \cos \theta_{3}$, etc.

Assuming that the gravity gradient torque is the only external disturbance torque acting along the pitch axis, we obtain the rotational equation of motion of a rigid body with an angular momentum $\vec{H}$ in a circular orbit as

$$
\left\{\frac{d \vec{H}}{d t}\right\}_{N} \equiv\left\{\frac{d \vec{H}}{d t}\right\}_{B}+\vec{\omega}^{B / N} \times \vec{H}=\vec{M}
$$

where $\{d / d t\}_{N}$ indicates differentiation with respect to time in reference frame $N$ and $\{d / d t\}_{B}$ indicates differentiation with respect to time in reference frame $B$. The gravity-gradient torque $\vec{M}$ is expressed in vector-dyadic form as:

$$
\vec{M}=3 n^{2} \vec{a}_{3} \times \hat{J} \cdot \vec{a}_{3}
$$

where $n=\sqrt{\mu / R_{c}^{3}}$ is the orbital rate, $\vec{a}_{3} \equiv-\vec{R}_{c} / R_{c}$, and $\hat{J}$ is the inertia dyadic of the spacecraft with respect to its mass center (Refs. 12-13).

Since $\vec{H}=\hat{J} \cdot \vec{\omega}^{B / N}$, the attitude dynamical equations of motion can be rewritten as

$$
\hat{J} \cdot \dot{\vec{\omega}}+\vec{\omega} \times \hat{J} \cdot \vec{\omega}=3 n^{2} \vec{a}_{3} \times \hat{J} \cdot \vec{a}_{3}
$$

where $\vec{\omega} \equiv \vec{\omega}^{B / N}$ and $\dot{\vec{\omega}}=\{d \vec{\omega} / d t\}_{N} \equiv\{d \vec{\omega} / d t\}_{B}$. Expressing $\vec{\omega}, \vec{a}_{3}$, and $\hat{J}$ in terms of basis vectors of the body-fixed reference frame $B$ as

$$
\begin{aligned}
\vec{\omega} & =\omega_{1} \vec{b}_{1}+\omega_{2} \vec{b}_{2}+\omega_{3} \vec{b}_{3} \\
\vec{a}_{3} & =C_{13} \vec{b}_{1}+C_{23} \vec{b}_{2}+C_{33} \vec{b}_{3} \\
\hat{J} & =\sum_{i=1}^{3} \sum_{j=1}^{3} J_{i j} \vec{b}_{i} \vec{b}_{j}
\end{aligned}
$$

we obtain the attitude dynamical equations of motion in matrix form as:

$$
\begin{array}{r} 
\\
\left.+\begin{array}{lll}
J_{11} & J_{12} & J_{13} \\
J_{21} & J_{22} & J_{23} \\
J_{31} & J_{32} & J_{33}
\end{array}\right]\left[\begin{array}{l}
\dot{\omega}_{1} \\
\dot{\omega}_{2} \\
\dot{\omega}_{3}
\end{array}\right] \\
+\left[\begin{array}{ccc}
0 & -\omega_{3} & \omega_{2} \\
\omega_{3} & 0 & -\omega_{1} \\
-\omega_{2} & \omega_{1} & 0
\end{array}\right]\left[\begin{array}{lll}
J_{11} & J_{12} & J_{13} \\
J_{21} & J_{22} & J_{23} \\
J_{31} & J_{32} & J_{33}
\end{array}\right]\left[\begin{array}{l}
\omega_{1} \\
\omega_{2} \\
\omega_{3}
\end{array}\right] \\
=3 n^{2}\left[\begin{array}{ccc}
0 & -C_{33} & C_{23} \\
C_{33} & 0 & -C_{13} \\
-C_{23} & C_{13} & 0
\end{array}\right]\left[\begin{array}{lll}
J_{11} & J_{12} & J_{13} \\
J_{21} & J_{22} & J_{23} \\
J_{31} & J_{32} & J_{33}
\end{array}\right]\left[\begin{array}{l}
C_{13} \\
C_{23} \\
C_{33}
\end{array}\right]
\end{array}
$$

The dynamical equations of motion about the bodyfixed principal axes become

$$
J_{1} \dot{\omega}_{1}-\left(J_{2}-J_{3}\right) \omega_{2} \omega_{3}=-3 n^{2}\left(J_{2}-J_{3}\right) C_{23} C_{33}
$$

$$
\begin{aligned}
& J_{2} \dot{\omega}_{2}-\left(J_{3}-J_{1}\right) \omega_{3} \omega_{1}=-3 n^{2}\left(J_{3}-J_{1}\right) C_{33} C_{13} \\
& J_{3} \dot{\omega}_{3}-\left(J_{1}-J_{2}\right) \omega_{1} \omega_{2}=-3 n^{2}\left(J_{1}-J_{2}\right) C_{13} C_{23}
\end{aligned}
$$

where

$$
\begin{aligned}
& C_{13}=-\sin \theta_{2} \cos \theta_{3} \\
& C_{23}=\cos \theta_{1} \sin \theta_{2} \sin \theta_{3}+\sin \theta_{1} \cos \theta_{2} \\
& C_{33}=-\sin \theta_{1} \sin \theta_{2} \sin \theta_{3}+\cos \theta_{1} \cos \theta_{2}
\end{aligned}
$$

for the sequence of $\mathbf{C}_{1}\left(\theta_{1}\right) \leftarrow \mathbf{C}_{3}\left(\theta_{3}\right) \leftarrow \mathbf{C}_{2}\left(\theta_{2}\right)$ under consideration. For this rotational sequence we have the following kinematic differential equations:

$$
\left[\begin{array}{c}
\dot{\theta}_{1} \\
\dot{\theta}_{2} \\
\dot{\theta}_{3}
\end{array}\right]=\frac{1}{\mathrm{c} \theta_{3}}\left[\begin{array}{ccc}
\mathrm{c} \theta_{3} & -\mathrm{c} \theta_{1} \mathrm{~s} \theta_{3} & \mathrm{~s} \theta_{1} \mathrm{~s} \theta_{3} \\
0 & \mathrm{c} \theta_{1} & -\mathrm{s} \theta_{1} \\
0 & \mathrm{~s} \theta_{1} \mathrm{c} \theta_{3} & \mathrm{c} \theta_{1} \mathrm{c} \theta_{3}
\end{array}\right]\left[\begin{array}{c}
\omega_{1} \\
\omega_{2} \\
\omega_{3}
\end{array}\right]+\left[\begin{array}{c}
0 \\
n \\
0
\end{array}\right]
$$

where $\mathrm{c} \theta_{i} \equiv \cos \theta_{i}$ and $\mathrm{s} \theta_{i} \equiv \sin \theta_{i}$.

One may linearize Eqs. (20)-(21) "about" an LVLH orientation while admitting a large pitch angle as follows. Assume $\theta_{1}$ and $\theta_{3}$ remain small, allow $\theta_{2}$ to be large, assume $\omega_{1}$ and $\omega_{3}$ are small, and $\omega_{2}$ is equal to the sum of a small quantity and $-n$. The attitude equations of motion that are linear in the small quantities can then be obtained as (Ref. 12):

$$
\begin{array}{r}
J_{1} \ddot{\theta}_{1}+\left(1+3 \cos ^{2} \theta_{2}\right) n^{2}\left(J_{2}-J_{3}\right) \theta_{1}-n\left(J_{1}-J_{2}+J_{3}\right) \dot{\theta}_{3} \\
\quad+3\left(J_{2}-J_{3}\right) n^{2}\left(\sin \theta_{2} \cos \theta_{2}\right) \theta_{3}=u_{1}+d_{1} \\
J_{2} \ddot{\theta}_{2}+3 n^{2}\left(J_{1}-J_{3}\right) \sin \theta_{2} \cos \theta_{2}=u_{2}+d_{2} \\
J_{3} \ddot{\theta}_{3}+\left(1+3 \sin ^{2} \theta_{2}\right) n^{2}\left(J_{2}-J_{1}\right) \theta_{3}+n\left(J_{1}-J_{2}+J_{3}\right) \dot{\theta}_{1} \\
\quad+3\left(J_{2}-J_{1}\right) n^{2}\left(\sin \theta_{2} \cos \theta_{2}\right) \theta_{1}=u_{3}+d_{3}
\end{array}
$$

where $u_{i}$ and $d_{i}$ are control and disturbance torques, respectively.

However, for a quasi-inertially stabilized, sun-pointing SSPS in geosynchronous orbit with small body rates, $\omega_{i}$ $(i=1,2,3)$, and small roll/yaw angles, $\theta_{1}$ and $\theta_{3}$, the kinematic differential equations, (21), can be linearized in the small quantities, as follows:

$$
\begin{aligned}
& \dot{\theta}_{1} \approx \omega_{1} \\
& \dot{\theta}_{2} \approx \omega_{2}+n \\
& \dot{\theta}_{3} \approx \omega_{3}
\end{aligned}
$$

The attitude equations of motion of a quasi-inertially stabilized, sun-pointing spacecraft with small roll and yaw angles, $\theta_{1}$ and $\theta_{3}$, can then be found as

$$
\begin{aligned}
J_{1} \ddot{\theta}_{1}= & -3 n^{2}\left(J_{2}-J_{3}\right)\left(\cos ^{2} \theta_{2}\right) \theta_{1} \\
& -3\left(J_{2}-J_{3}\right) n^{2}\left(\sin \theta_{2} \cos \theta_{2}\right) \theta_{3}+u_{1}+d_{1} \\
J_{2} \ddot{\theta}_{2}= & -3 n^{2}\left(J_{1}-J_{3}\right) \sin \theta_{2} \cos \theta_{2}+u_{2}+d_{2} \\
J_{3} \ddot{\theta}_{3}= & -3 n^{2}\left(J_{2}-J_{1}\right)\left(\sin ^{2} \theta_{2}\right) \theta_{3} \\
& -3\left(J_{2}-J_{1}\right) n^{2}\left(\sin \theta_{2} \cos \theta_{2}\right) \theta_{1}+u_{3}+d_{3}
\end{aligned}
$$


Node Number Locations for Normal Modes Results

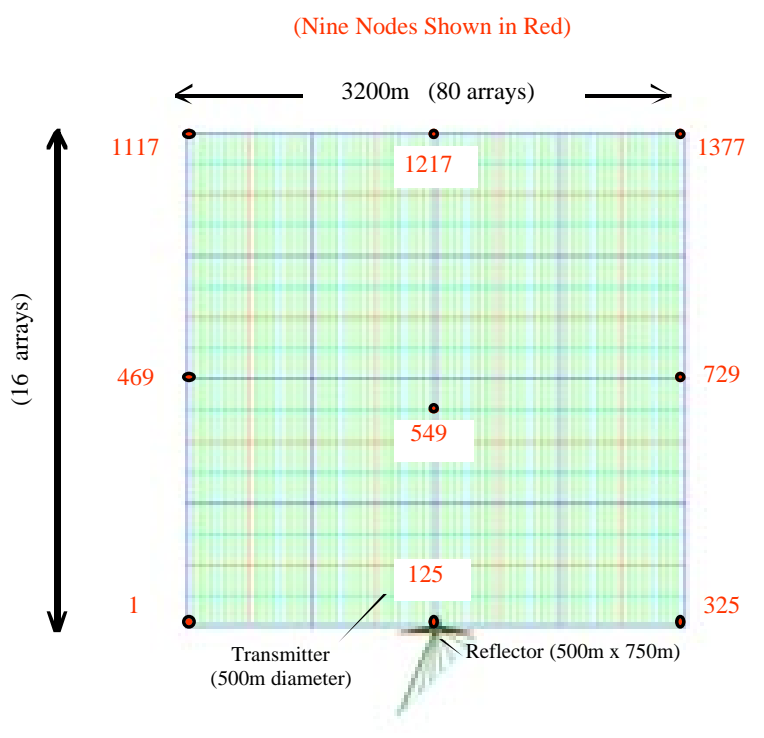

Front View (Abaqus support truss in back)

Figure 5: Selected FEM node locations for control analysis and design (Courtesy of Tim Collins at NASA LaRC).

The pitch attitude angle relative to the LVLH frame, $\theta_{2}$, is not restricted to be small, but it may be regarded as a sum, $\theta_{2}=n t+\delta \theta_{2}$, where $\delta \theta_{2}$ is a small pitch attitude error. Kinematical and dynamical differential equations can then be made linear in the small quantities $\omega_{1}, \omega_{2}$, $\omega_{3}, \theta_{1}, \delta \theta_{2}$, and $\theta_{3}$. For such a case, Eqs. (21) become

$$
\dot{\theta}_{1} \approx \omega_{1} ; \quad \delta \dot{\theta}_{2} \approx \omega_{2} ; \quad \dot{\theta}_{3} \approx \omega_{3}
$$

and Eqs. (24) become

$$
\begin{array}{r}
J_{1} \ddot{\theta}_{1}+3 n^{2}\left(J_{2}-J_{3}\right)\left[\left(\cos ^{2} n t\right) \theta_{1}+(1 / 2)(\sin 2 n t) \theta_{3}\right] \\
=u_{1}+d_{1} \\
J_{2} \delta \ddot{\theta}_{2}+3 n^{2}\left(J_{1}-J_{3}\right)\left[\left(\cos ^{2} n t-\sin ^{2} n t\right) \delta \theta_{2}\right. \\
+(1 / 2) \sin 2 n t]=u_{2}+d_{2} \\
J_{3} \ddot{\theta}_{3}+3 n^{2}\left(J_{2}-J_{1}\right)\left[\left(\sin ^{2} n t\right) \theta_{3}+(1 / 2)(\sin 2 n t) \theta_{1}\right] \\
=u_{3}+d_{3}
\end{array}
$$

where $\left(\theta_{1}, \delta \theta_{2}, \theta_{3}\right)$ are the small roll, pitch, and yaw attitude errors of a sun-pointing spacecraft, respectively.

Equations (24) or (26) are the attitude equations of motion of the Abacus satellite for control design in the presence of the external disturbances, $d_{i}$, described as in Eqs. (1).

\section{$5 \quad$ Structural Dynamic Models}

Dynamics and control problems of large flexible platforms in space, such as the square Abacus satellite, have been investigated by many researchers in the past. The flexible structural dynamics and control problem is a
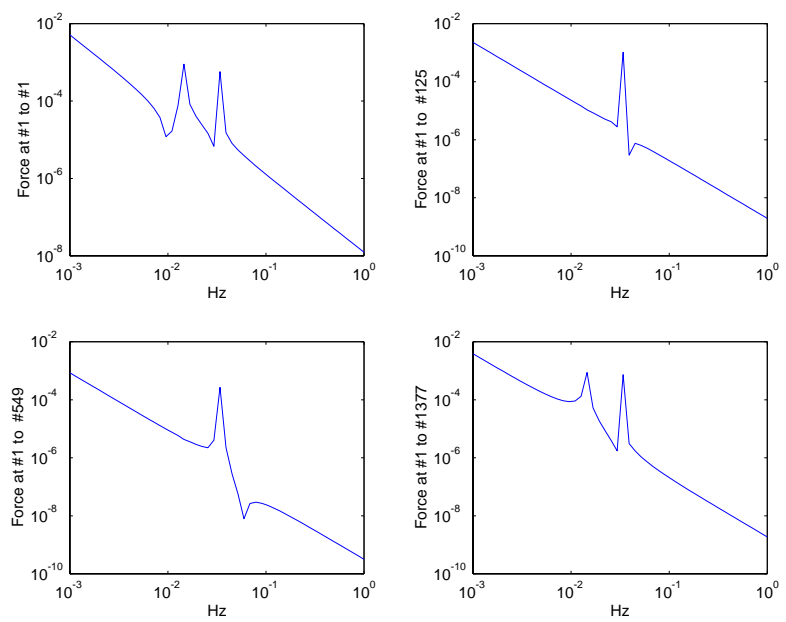

Figure 6: Bode magnitude plots of reduced-order transfer functions from an input force at node \#1 to various output locations.

topic of continuing practical as well as theoretical interest. However, a significant control-structure interaction problem, possible for such a very large Abacus platform $(3.2 \times 3.2 \mathrm{~km})$ with the lowest structural mode frequency of about $0.002 \mathrm{~Hz}$, is avoided simply by designing an attitude control system with very low bandwidth $(<$ orbit frequency of $1 \times 10^{-5} \mathrm{~Hz}$ ). The proposed low-bandwidth attitude control system (to be presented in the next section), however, utilizes a concept of cyclic-disturbance accommodation control to provide the required \pm 5 arcmin pointing accuracy of the Abacus platform in the presence of large, but slowly varying, external disturbances and dynamic modeling uncertainties. Consequently, the flexible structural control problem is not further elaborated in this study, while a structural dynamic interaction problem with thermal distortion needs to be further investigated in a future study.

Various structural concepts for providing the required stiffness and rigidity of the Abacus platform have been presented in Refs $4-5$. Selected node locations for control analysis and design are shown in Figure 5. Typical pole-zero patterns of reduced-order transfer functions can be seen in Figure 6. Computer simulation results of a reduced-order structural model with the lowest 16 modes, confirm that the control-structure interaction problem can be simply avoided by a low-bandwidth attitude control system.

\section{Control System Architecture}

The area-to-mass ratio of the Abacus satellite, $A / m=$ $0.4 \mathrm{~m}^{2} / \mathrm{kg}$, relatively large when compared to $0.02 \mathrm{~m}^{2} / \mathrm{kg}$ of typical geosynchronous communications satellites, is a key parameter characterizing the very large size of the Abacus satellite. If left uncontrolled, this can cause a cyclic drift in the longitude of the Abacus satellite of 2 
deg, east and west. Thus, in addition to standard northsouth and east-west stationkeeping maneuvers for \pm 0.1 deg orbit position control, active control of the orbit eccentricity using ion thrusters with high specific impulse, $I_{s p}$, becomes mandatory. Furthermore, continuous sun tracking of the Abacus satellite requires large control torques to counter various disturbance torques. A control system architecture developed in this study utilizes properly distributed ion thrusters to counter, simultaneously, the cyclic pitch gravity-gradient torque, and solar radiation pressure.

\subsection{Electric Propulsion System}

Basic characteristics of an electric propulsion system for the Abacus satellite are summarized in Table 5. Approximately $85,000 \mathrm{~kg}$ of propellant per year is required for simultaneous orbit, attitude, and structural control using a minimum of 500 1-N electric propulsion thrusters with $I_{s p}=5,000 \mathrm{sec}$. The yearly propellant requirement is reduced to $21,000 \mathrm{~kg}$ if an $I_{s p}$ of $20,000 \mathrm{sec}$ can be achieved (as was assumed for the 1979 SSPS reference system). As $I_{s p}$ is increased, the propellant mass decreases but the electric power requirement increases; consequently, the mass of solar arrays and power processing units increases. Based on a minimum of $5001-\mathrm{N}$ thrusters, a mass/power ratio of $5 \mathrm{~kg} / \mathrm{kW}$, and a power/thrust ratio of $30 \mathrm{~kW} / \mathrm{N}$, the total dry mass (power processing units, thrusters, tanks, feed systems, etc.) of an electric propulsion system proposed for the Abacus satellite is estimated as $75,000 \mathrm{~kg}$.

The capability of present electric thrusters is orders of magnitude below that required for the Abacus satellite. If the xenon fueled, 1-kW level, off-the-shelf ion engines available today are to be employed, the number of thrusters would be increased to 15,000 . The actual total number of ion engines will further increase significantly when we consider the ion engine's lifetime, reliability, duty cycle, and redundancy.

For example, the $2.3-\mathrm{kW}, 30-\mathrm{cm}$ diameter ion engine of the Deep Space 1 spacecraft has a maximum thrust level of $92 \mathrm{mN}$. Throttling down is achieved by feeding less electricity and xenon propellant into the propulsion system. Specific impulse ranges from 1,900 sec at the minimum throttle level to $3,200 \mathrm{sec}$.

In principle, an electric propulsion system employs electrical energy to accelerate ionized particles to extremely high velocities, giving a large total impulse for a small consumption of propellant. In contrast to standard propulsion, in which the products of chemical combustion are expelled from a rocket engine, ion propulsion is accomplished by giving a gas, such as xenon (which is like neon or helium, but heavier), an electrical charge and electrically accelerating the ionized gas to a speed of about $30 \mathrm{~km} / \mathrm{s}$. When xenon ions are emitted at such high speed as exhaust from a spacecraft, they push the spacecraft in the opposite direction. However, the ex-
Table 5: Electric propulsion system for the 1.2-GW Abacus satellite

\begin{tabular}{ll}
\hline Thrust, $T$ & $\geq 1 \mathrm{~N}$ \\
Specific impulse, $I_{s p}=T /(\dot{m} g)$ & $\geq 5,000 \mathrm{sec}$ \\
Exhaust velocity, $V_{e}=I_{s p} g$ & $\geq 49 \mathrm{~km} / \mathrm{s}$ \\
Total efficiency, $\eta=P_{o} / P_{i}$ & $\geq 80 \%$ \\
Power/thrust ratio, $P_{i} / T$ & $\leq 30 \mathrm{~kW} / \mathrm{N}$ \\
Mass/power ratio & $\leq 5 \mathrm{~kg} / \mathrm{kW}$ \\
Total peak thrust & $200 \mathrm{~N}$ \\
Total peak power & $6 \mathrm{MW}$ \\
Total average thrust & $80 \mathrm{~N}$ \\
Total average power & $2.4 \mathrm{MW}$ \\
Number of 1-N thrusters & $\geq 500$ \\
Total dry mass & $\geq 75,000 \mathrm{~kg}$ \\
Propellant consumption & $85,000 \mathrm{~kg} /$ year \\
\hline
\end{tabular}

Note: $T=\dot{m} V_{e}, P_{o}=\frac{1}{2} \dot{m} V_{e}^{2}=\frac{1}{2} T V_{e}, P_{o} / T=\frac{1}{2} V_{e}=$ ideal power/thrust ratio, $P_{i} / T=\frac{1}{2 \eta} V_{e}, I_{s p}=T /(\dot{m} g)=$ $V_{e} / g, V_{e}=I_{s p} g$ where $g=9.8 \mathrm{~m} / s^{2}, \dot{m}$ is the exhaust mass flow rate, $P_{i}$ is the input power, and $P_{o}$ is the output power.

haust gas from an ion thruster consists of large numbers of positive and negative ions that form an essentially neutral plasma beam extending for large distances in space. It seems that little is known yet about the long-term effect of such an extensive plasma on geosynchronous satellites with regard to communications, solar cell degradation, environmental contamination, etc.

\subsection{Control System Architecture}

A control system architecture developed in this study is shown in Figure 7. The proposed control system utilizes properly distributed ion thrusters to counter, simultaneously, the cyclic pitch gravity-gradient torque, the secular roll torque caused by an offset of the center-of-mass and center-of-pressure, the cyclic roll/yaw microwave radiation torque, and the solar radiation pressure force whose average value is about $60 \mathrm{~N}$.

A significant control-structure interaction problem, possible for such very large Abacus platform with the lowest structural mode frequency of $0.002 \mathrm{~Hz}$, is simply avoided by designing an attitude control system with very low bandwidth ( $<$ orbit frequency). However, the proposed low-bandwidth attitude control system utilizes a concept of cyclic-disturbance accommodating control to provide \pm 5 arcmin pointing of the Abacus platform in the presence of large, but slowly varying, external disturbances and dynamic modeling uncertainties.

The concept of cyclic-disturbance accommodation control has been successfully applied to a variety of space vehicle control problems (Ref. 12), including the Hubble Space Telescope, the International Space Station, flexible space structures, and halo orbit control. High-bandwidth, colocated direct velocity feedback, ac- 


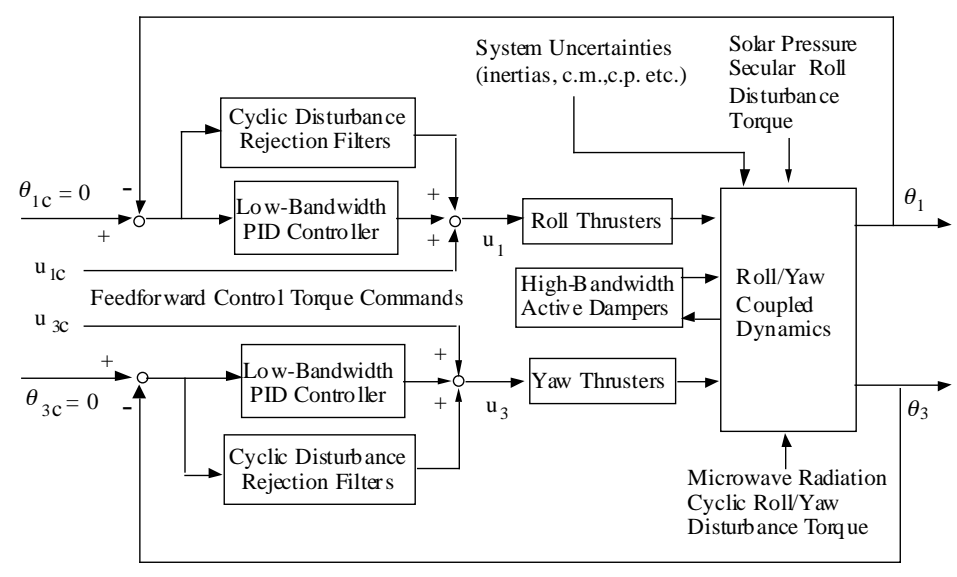

Feed forward Control Torque Command

$\mathrm{u}_{2 \mathrm{c}}=3 \mathrm{n}^{2}\left(\overline{\mathrm{J}}_{1}-\overline{\mathrm{J}}_{3}\right)(\sin 2 \mathrm{nt}) / 2$

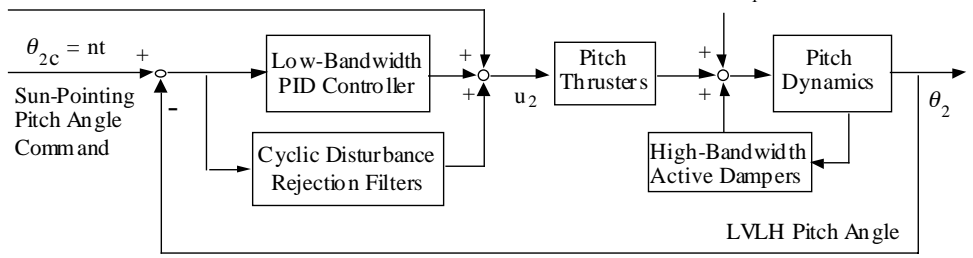

Figure 7: An integrated orbit, attitude, and structural control system architecture employing electric propulsion thrusters.

tive dampers may need to be properly distributed over the platform. Detailed technical discussions of practical control systems design for space vehicles in the presence structural flexibility as well as persistent external disturbances can be found in Ref. 12. Thus, theoretical aspects of the control law design problem of the Abacus satellite are not elaborated upon in this paper.

Placement of approximately $5001-\mathrm{N}$ electric propulsion thrusters at 12 different locations is illustrated in Figure 8.

In contrast to a typical placement of thrusters at the four corners, e.g., employed for the 1979 SSPS reference system, the proposed placement shown in Figure 8 minimizes roll/pitch thruster couplings as well as the excitation of platform out-of-plane structural modes. A minimum of 500 ion engines of 1-N thrust level are required for simultaneous attitude and stationkeeping control. When reliability, lifetime, duty cycle, lower thrust level, and redundancy of ion engines are considered, this number will increase significantly.

\subsection{Control Simulation Results}

Control system simulation results of an illustrative case with 10-deg initial attitude errors are shown in Figure 9 . In this simulation, various dynamic modeling uncertainties (i.e., $\pm 20 \%$ uncertainties for the moments and products of inertia, cm-cp offset, external disturbances, etc.) are included. The proposed low-bandwidth

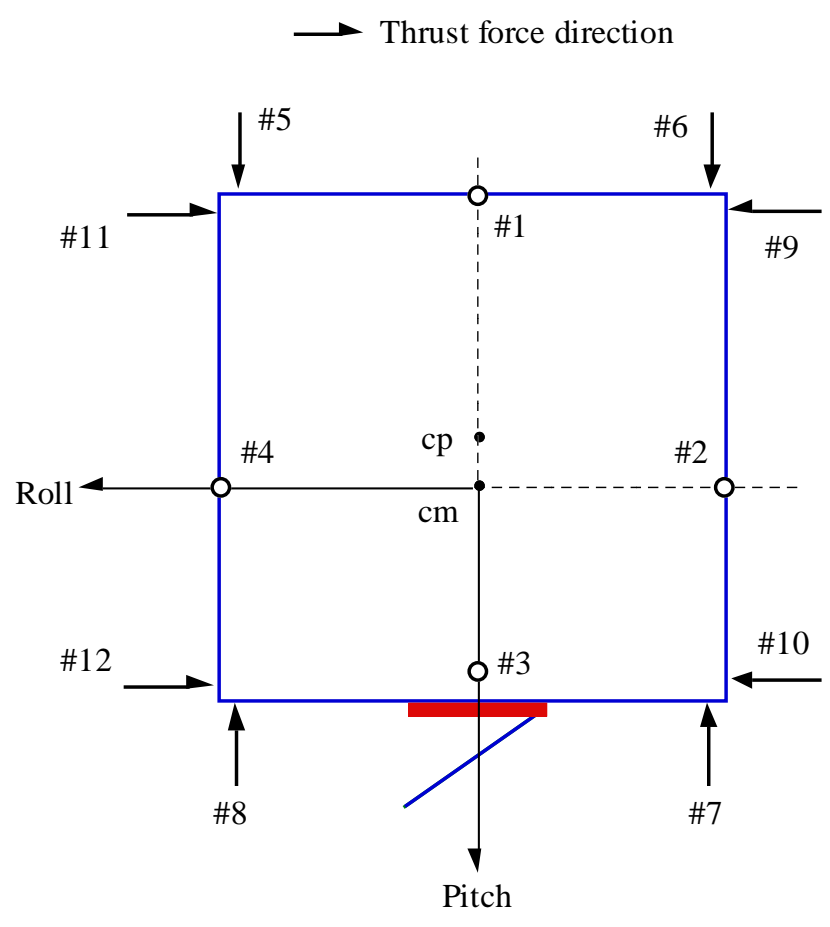

Roll: $1 / 3 \quad$ Pitch: $2 / 4 \quad$ Yaw. 5/6/7/8

Orbit Eccentricity, Roll/Pitch Control: 1/3, 2/4

E/W and Yaw Control: 9/10/11/12

N/S and Yaw Control: 5/6/7/8

Figure 8: Placement of a minimum of $5001-\mathrm{N}$ electric propulsion thrusters at 12 different locations, with 100 thrusters each at locations \#2 and \#4.
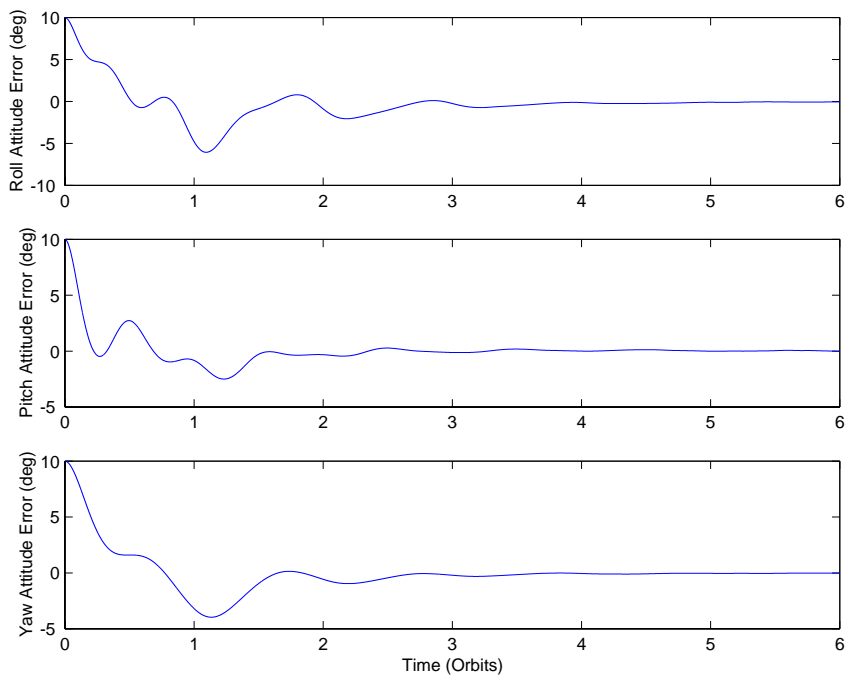

Figure 9: Control simulation results for the proposed attitude control system architecture. 

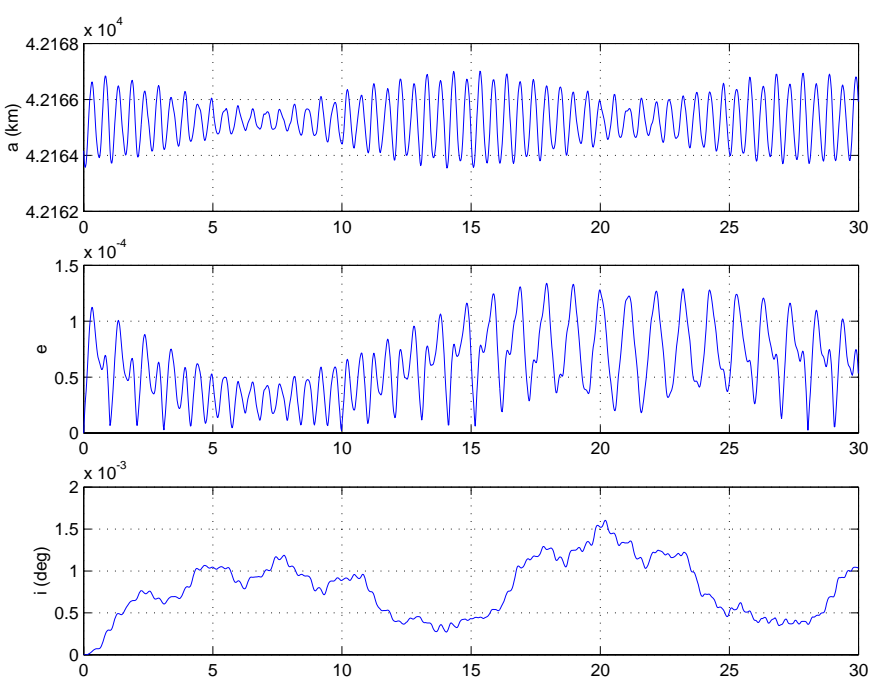

Figure 10: Orbit control simulation results with continuous (non-impulsive) eccentricity and inclination control (time in units of orbits).

attitude control system, which effectively utilizes the concept of cyclic-disturbance accommodation control, maintains the required \pm 5 arcmin, steady-state pointing of the Abacus platform in the presence of large, but slowly varying, external disturbances and dynamic modeling uncertainties. The total thrusting force from the roll/pitch thrusters \#1 through \#4 nearly counters the $60-\mathrm{N}$ solar radiation pressure force; however, any residual $\Delta V$ caused by various uncertainties should be corrected during standard east-west stationkeeping maneuvers.

Orbit control simulation results with the effects of earth's oblateness and triaxiality, luni-solar perturbations, 60-N solar radiation pressure force, and simultaneous orbit and attitude control thruster firings are shown in Figure 10. It can be seen that the eccentricity and inclination are all properly maintained. The feasibility of using continuous (non-impulsive) firings of ion thrusters for simultaneous attitude and orbit control is demonstrated in this study; however, a further detailed orbit control study is needed. The orbit control problem of geosynchronous satellites is a topic of continuing practical interest (Refs. 14-16).

\section{Summary and Recommenda- tions}

\subsection{Summary of Study Results}

Despite the importance of the cyclic pitch gravitygradient torque, this study has shown that the solar radiation pressure force is considerably more detrimental to control of the Abacus satellite (and also other large SSPS) because of an area-to-mass ratio that is very large compared to contemporary, higher-density spacecraft.

A key parameter that characterizes the Abacus satellite is its area-to-mass ratio, $A / m$, of $0.4 \mathrm{~m}^{2} / \mathrm{kg}$, which is relatively large when compared to $0.02 \mathrm{~m}^{2} / \mathrm{kg}$ for typical geosynchronous communications satellites.

A significant control-structure interaction problem, possible for such very large, flexible Abacus platform with the lowest structural mode frequency of $0.002 \mathrm{~Hz}$, is simply avoided by designing an attitude control system with very low bandwidth (< orbit frequency). However, the proposed low-bandwidth attitude control system effectively utilizes a concept of cyclic-disturbance accommodating control to provide \pm 5 arcmin pointing of the Abacus platform in the presence of large, but slowly varying, external disturbances and dynamic modeling uncertainties.

Approximately 85,000 kg of propellant per year is required for simultaneous orbit, attitude, and structural control using a minimum of 500 1-N electric propulsion thrusters with $I_{s p}=5,000$ sec. The total dry mass (power processing units, thrusters, tanks, feed systems, etc.) of an electric propulsion system proposed for the Abacus satellite is estimated as $75,000 \mathrm{~kg}$.

\subsection{Recommendations for Future Re- search}

The baseline control system architecture developed for the Abacus satellite requires a minimum of 500 ion engines of 1-N thrust level. The capability of present electric thrusters is orders of magnitude below that required for the Abacus satellite. If the xenon fueled, 1-kW level, off-the-shelf ion engines available today, are to be employed, the number of thrusters would be increased to 15,000. The actual total number of ion engines will further increase significantly when we consider the ion engine's lifetime, reliability, duty cycle, redundancy, etc. Consequently, a $30-\mathrm{kW}, 1-\mathrm{N}$ level electric propulsion thruster with a specific impulse greater than 5,000 sec needs to be developed for the Abacus satellite if an excessively large number of thrusters is to be avoided.

Several high-power electric propulsion systems are currently under development. For example, the NASA T-220 10-kW Hall thruster recently completed a 1,000hr life test. This high-power (over $5 \mathrm{~kW}$ ) Hall thruster provides $500 \mathrm{mN}$ of thrust at a specific impulse of 2,450 sec and 59\% total efficiency. Dual-mode Hall thrusters, which can operate in either high-thrust mode or high$I_{s p}$ mode for efficient propellant usage, are also being developed.

The exhaust gas from an electric propulsion system forms an essentially neutral plasma beam extending for large distances in space. Because little is known yet about the long-term effect of an extensive plasma on geosynchronous satellites with regard to communications, solar cell degradation, etc, the use of lightweight, space-assembled large-diameter momentum wheels may 
Table 6: Technology advancement needs for the Abacus SSPS

\begin{tabular}{lc}
\hline Electric Thrusters & $30 \mathrm{~kW}, 1 \mathrm{~N}$ \\
& $I_{s p}>5000 \mathrm{sec}$ \\
& $(500-1,000$ thrusters $)$ \\
\hline CMGs & $2,000 \mathrm{~N}-\mathrm{m}-\mathrm{s} / \mathrm{kg}$ \\
& $500,000 \mathrm{~N}-\mathrm{m}-\mathrm{s} / \mathrm{unit}$ \\
\hline Space-Assembled & $66,000 \mathrm{~N}-\mathrm{m}-\mathrm{s} / \mathrm{kg}$ \\
Momentum Wheels & $4 \times 10^{8} \mathrm{~N}-\mathrm{m}-\mathrm{s} / \mathrm{unit}$ \\
& $(5-10 \mathrm{MWs})$ \\
\hline
\end{tabular}

also be considered as an option for the Abacus satellite; therefore, these devices warrant further study. In Table 6 , the technology advances required for the Abacus satellite are summarized. It is emphasized that both electric propulsion and momentum wheel technologies require significant advancement to support the development of large SSPS.

Despite the huge size and low structural frequencies of the Abacus satellite, the control-structure interaction problem appears to be a tractable one because the tight pointing control requirement can be met even with a control bandwidth that is much lower than the lowest structural frequency. However, further detailed study needs to be performed for achieving the required 5arcmin microwave beam pointing accuracy in the presence of transmitter/reflector-coupled structural dynamics, Abacus platform thermal distortion and vibrations, hardware constraints, and other short-term impulsive disturbances.

Although the rotating reflector concept of the Abacus satellite eliminates massive rotary joint and slip rings of the 1979 SSPS reference concept, the transmitter fixed to the Abacus platform results in unnecessarily tight pointing requirements imposed on the platform. Further system-level tradeoffs will be required for the microwavetransmitting antenna design, such as whether or not to gimbal it with respect to the platform, use mechanical or electronic beam steering, and so forth.

\section{Acknowledgment}

This paper is based on NASA CR of NASA contract NAS1-00122 which was monitored by Jessica WoodsVedeler and Chris Moore at NASA Langley Research Center. The authors would like to thank the SSP (Space Solar Power) Exploratory Research and Technology program of NASA for supporting this project. In particular, the authors are indebted to Connie Carrington, Harvey Feingold, Chris Moore, and John Mankins without whose previous SSP systems engineering work this dynamics \& control research would not have been possible. Special thanks also go to Jessica Woods-Vedeler and Tim Collins at NASA Langley Research Center for their technical support and guidance throughout the course of this study.

\section{References}

[1] Glaser, P. E., "Power from the Sun: Its Future," Science, Vol. 162, No. 3856, November 22, 1968, pp. 857-861.

[2] Glaser, P. E., "The Potential of Satellite Solar Power," Proceedings of the IEEE, Vol. 65, No. 8, August 1977, pp. 1162-1176.

[3] Mankins, J. C., "A Fresh Look at Space Solar Power: New Architecture, Concepts, and Technologies," IAF-97-R.2.03, the 48th International Astronautical Congress, Turin, Italy, October 6-10, 1997.

[4] Moore, C., "Structural Concepts for Space Solar Power Satellites," SSP Systems Workshop, NASA Glenn Research Center, September 8, 1999.

[5] Moore, C., "Structures, Materials, Controls and Thermal Management," SSP Technical Interchange Meeting \#3, Huntsville, AL, June 19-23, 2000.

[6] Carrington, C. and Feingold, H., "SSP Systems Integration, Analysis and Modeling," SSP Technical Interchange Meeting \#3, Huntsville, AL, June 19$23,2000$.

[7] Oglevie, R. E., "Attitude Control of Large Solar Power Satellites," Proceedings of AIAA Guidance and Control Conference, Palo Alto, CA, 1978, pp. $571-578$.

[8] Elrod, B. D., "A Quasi-Inertial Attitude Mode for Orbiting Spacecraft," Journal of Spacecraft and Rockets, Vol. 9, December, 1972, pp. 889-895.

[9] Juang, J.-N. and Wang, S.-J., "An Investigation of Quasi-Inertial Attitude Control for a Solar Power Satellite," Space Solar Power Review, Vol. 3, 1982, pp. 337-352.

[10] McInnes, C. R., Solar Sailing: Technology, Dynamics and Mission Applications, Springer Praxis Publishing, Chichester, UK, 1999.

[11] Agrawal, B. N., Design of Geosynchronous Spacecraft, Englewood Cliffs, NJ, Prentice-Hall, 1986.

[12] Wie, B., Space Vehicle Dynamics and Control, AIAA Education Series, AIAA, Washington, DC, 1998.

[13] Roithmayr, C. M., "Gravitational Moment Exerted on a Small Body by an Oblate Body," Journal of Guidance, Control, and Dynamics, Vol. 12, No. 3, 1989, pp. 441-444.

[14] Gartrell, C. F., "Simultaneous Eccentricity and Drift Rate Control," Journal of Guidance and Control, Vol. 4, No. 3, 1981, pp. 310-315.

[15] Kamel, A. A. and Wagner, C. A., "On the Orbital Eccentricity Control of Synchronous Satellites," Journal of the Astronautical Sciences, Vol. XXX, No. 1, 1982, pp. 61-73.

[16] Kelly, T. J., White, L. K., and Gamble, D. W., "Stationkeeping of Geostationary Satellites with Simultaneous Eccentricity and Longitudinal Control," Journal of Guidance, Control and Dynamics, Vol. 17, No. 4, 1994, pp. 769-777. 